\title{
CANDIDATE CLUSTERS OF GALAXIES AT $z>1.3$ IDENTIFIED IN THE SPITZER SOUTH POLE TELESCOPE DEEP FIELD SURVEY
}

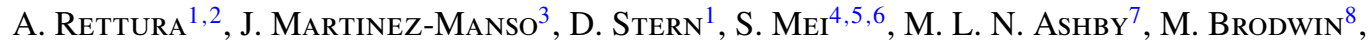 \\ D. GetTings ${ }^{3}$, A. H. Gonzalez ${ }^{3}$, S. A. STANFord ${ }^{9,10}$, AND J. G. BartLetT ${ }^{11}$ \\ ${ }^{1}$ Jet Propulsion Laboratory, California Institute of Technology, MS 169-234, Pasadena, CA 91109, USA \\ 2 Department of Astronomy, California Institute of Technology, MS 249-17, Pasadena, CA 91125, USA \\ ${ }^{3}$ Department of Astronomy, University of Florida, Gainesville, FL 32611, USA \\ ${ }^{4}$ GEPI, Observatoire de Paris, Section de Meudon, Meudon Cedex, France \\ ${ }^{5}$ University of Paris Denis Diderot, 75205 Paris Cedex 13, France \\ ${ }^{6}$ Infrared Processing and Analysis Center, Pasadena, CA 91125, USA \\ ${ }^{7}$ Harvard-Smithsonian Center for Astrophysics, Cambridge, MA 02138, USA \\ ${ }^{8}$ Department of Physics and Astronomy, University of Missouri, Kansas City, MO 64110, USA \\ ${ }^{9}$ Department of Physics, University of California, Davis, CA 95616, USA \\ ${ }^{10}$ Institute of Geophysics and Planetary Physics, Lawrence Livermore National Laboratory, Livermore, CA 94551, USA \\ ${ }^{11}$ APC, AstroParticule et Cosmologie, Universite Paris Diderot, CNRS/IN2P3, CEA/lrfu, Observatoire de Paris, \\ Sorbonne Paris Cite, 75205 Paris Cedex 13, France \\ Received 2014 March 20; accepted 2014 October 14; published 2014 December 5
}

\begin{abstract}
We present 279 galaxy cluster candidates at $z>1.3$ selected from the $94 \mathrm{deg}^{2}$ Spitzer South Pole Telescope Deep Field (SSDF) survey. We use a simple algorithm to select candidate high-redshift clusters of galaxies based on Spitzer/IRAC mid-infrared data combined with shallow all-sky optical data. We identify distant cluster candidates adopting an overdensity threshold that results in a high purity (80\%) cluster sample based on tests in the Spitzer Deep, Wide-Field Survey of the Boötes field. Our simple algorithm detects all three $1.4<z \leqslant 1.75 \mathrm{X}$-ray detected clusters in the Boötes field. The uniqueness of the SSDF survey resides not just in its area, one of the largest contiguous extragalactic fields observed with Spitzer, but also in its deep, multi-wavelength coverage by the South Pole Telescope (SPT), Herschel/SPIRE, and XMM-Newton. This rich data set will allow direct or stacked measurements of Sunyaev-Zel'dovich effect decrements or X-ray masses for many of the SSDF clusters presented here, and enable a systematic study of the most distant clusters on an unprecedented scale. We measure the angular correlation function of our sample and find that these candidates show strong clustering. Employing the COSMOS/UltraVista photometric catalog in order to infer the redshift distribution of our cluster selection, we find that these clusters have a comoving number density $n_{c}=\left(0.7_{-0.6}^{+6.3}\right) \times 10^{-7} h^{3} \mathrm{Mpc}^{-3}$ and a spatial clustering correlation scale length $r_{0}=(32 \pm 7) h^{-1}$ Mpc. Assuming our sample is comprised of dark matter halos above a characteristic minimum mass, $M_{\min }$, we derive that at $z=1.5$ these clusters reside in halos larger than $M_{\min }=1.5_{-0.7}^{+0.9} \times 10^{14} h^{-1} M_{\odot}$. We find that the mean mass of our cluster sample is equal to $M_{\text {mean }}=1.9_{-0.8}^{+1.0} \times 10^{14} h^{-1} M_{\odot}$; thus, our sample contains the progenitors of present-day massive galaxy clusters.
\end{abstract}

Key words: cosmology: observations - galaxies: clusters: general - galaxies: high-redshift - galaxies: statistics infrared: galaxies - large-scale structure of universe

Online-only material: color figures

\section{INTRODUCTION}

Emerging from the cosmic web, galaxy clusters are the most massive gravitationally bound structures in the universe. Thought to have begun their assembly at $z>2$, clusters provide insights into the growth of large-scale structure as well as the physics that drives galaxy evolution. How and when the most massive galaxies assemble their stellar mass, stop forming stars, and acquire their observed morphologies remain outstanding questions. The redshift range $1.4<z<2$ is a key epoch in this respect: elliptical galaxies start to become the dominant population in cluster cores, and star formation in spiral galaxies is being quenched (e.g., Blakeslee et al. 2006; Rosati et al. 2009; Mei et al. 2009; Overzier et al. 2009; Rettura et al. 2010, 2011; Raichoor et al. 2011; Strazzullo et al. 2010; Stanford et al. 2012; Snyder et al. 2012; Zeimann et al. 2012; Mei et al. 2012; Nantais et al. 2013). Interestingly, some field galaxy studies find that the star formation rate (SFR)-density relation reverses at $z=1$ relative to $z=0$ (Cooper et al. 2007; Elbaz et al. 2007), such that star formation no longer decreases with increasing galaxy density at $z=1$. However, other studies disagree with this result (Patel et al. 2009; Muzzin et al. 2012; Scoville et al. 2013) and conclude that the reversal must happen at $z>1$, as they find the local density correlations to be already in place by $z=1$. There is also observational evidence for a progressive increase in the amount of star formation that occurs in galaxy cluster cores at $z \gtrsim 1.4$ (e.g., Hilton et al. 2009; Hayashi et al. 2010; Tran et al. 2010; Fassbender et al. 2011; Tadaki et al. 2012; Brodwin et al. 2013; Alberts et al. 2014). This suggests that significant star formation is occurring in high-density environments at early epochs. Therefore, increasing evidence points to clusters at $1.5<z<2$ as being the ideal laboratories to study cluster formation and to catch transformations in their stellar populations.

Until recently, however, this redshift range was essentially unreachable with the available instrumentation, with clusters at these redshifts being exceedingly challenging to identify from either ground-based optical/near-infrared (NIR) imaging 
or from X-ray surveys. Mid-infrared (MIR) imaging with Spitzer has changed the landscape. Previous Spitzer wide-area surveys have proven effective at identifying samples of galaxy clusters down to low masses at $1 \lesssim z<2$ (e.g., SDWFS, SWIRE, CARLA; Eisenhardt et al. 2008; Papovich 2008; Wilson et al. 2009; Demarco et al. 2010; Galametz et al. 2010; Stanford et al. 2012; Zeimann et al. 2012; Brodwin et al. 2013; Galametz et al. 2013; Muzzin et al. 2013b; Wylezalek et al. 2013), where current X-ray observations are restricted to only the most massive systems. X-ray follow-up has verified several of these MIR-selected clusters, implying masses of a few $10^{14} M_{\odot}$ (Papovich et al. 2010; Brodwin et al. 2011; Muzzin et al. 2013b). To date, however, only a few clusters have been confirmed at $z>1.5$, in part due to a lack of sufficiently large Spitzer surveys.

With the Spitzer South Pole Telescope Deep Field survey (SSDF; Ashby et al. 2013), we aim to discover hundreds of cluster candidates at these redshifts. The uniqueness of the SSDF survey resides not just in its area, $94 \mathrm{deg}^{2}$, one of the largest contiguous extragalactic fields surveyed with Spitzer, but also in its coverage, with deep observations for the Sunyaev-Zel'dovich (SZ) effect by the South Pole Telescope (SPT), and with even deeper observations being taken with the new SPT camera, SPTpol (George et al. 2012). Approximately one-fourth of the SSDF field also has deep X-ray observations from the XMMNewton XXL Survey (Pierre et al. 2011). This rich multiwavelength data set will allow us to determine cluster masses for many of the SSDF clusters at $1.5<z<2$, enabling a systematic study of the cluster population at an important cosmic epoch.

The structure of this paper is as follows. The description of our data sets comprises Section 2. In Section 3, we describe the method we employ to identify distant galaxy clusters, and we estimate our sample purity based on analysis of the Boötes field. In Section 4, we study the clustering of our sample, deriving the characteristic minimum mass, $M_{\min }$, of the dark matter halos in which our clusters reside. Section 5 summarizes the conclusions of our study. Throughout, we assume a $\Omega_{\Lambda}=0.73, \Omega_{m}=0.27$, and $H_{0}=71 \mathrm{~km} \mathrm{~s}^{-1} \mathrm{Mpc}^{-1}$ cosmology (Spergel et al. 2003), and use magnitudes in the AB system.

\section{THE SPITZER SOUTH POLE TELESCOPE DEEP FIELD SURVEY}

The SSDF, centered at $23^{\mathrm{h}} 30^{\mathrm{m}},-55^{\mathrm{d}} 00^{\mathrm{m}}(\mathrm{J} 2000)$, is a widearea survey using the Spitzer Infrared Array Camera (IRAC; Fazio et al. 2004) to cover $94 \mathrm{deg}^{2}$ of extragalactic sky. We discuss the IRAC (Section 2.1) and publicly available optical data (Section 2.2) next. Ashby et al. (2013) summarizes other available data in this field. This includes X-ray observations from XMM-Newton as part of the XXL survey (Pierre et al. 2011), of which a $25 \mathrm{deg}^{2}$ portion is located within the SSDF. Covering the entire SSDF region are shallow near-infrared data from the VISTA Hemisphere Survey (VHS) in the $J, H$, and $K$ bands that reach $5 \sigma$ depths of $12.0,17.4$, and $20.2 \mu \mathrm{Jy}$, respectively (R. McMahon et al., in preparation); far-infrared data from Herschel/SPIRE at 250, 350, and $500 \mu \mathrm{m}$ that reach $1 \sigma$ depths (per beam) of $10 \mathrm{mJy}$ in all three bands (Holder et al. 2013); and millimeter data from the SPT telescope at 1400, 2000 , and $3000 \mu \mathrm{m}$ that reach $5 \sigma$ depths of 15,5 , and $10 \mathrm{mJy}$, respectively (Carlstrom et al. 2011; Austermann et al. 2012; Story et al. 2013). In addition, the Blanco Cosmology Survey (BCS) surveyed $\sim 30 \mathrm{deg}^{2}$ of the SSDF in the griz bands down to 23 mag (AB; 10 $\sigma$; Desai et al. 2012; Bleem et al. 2014) and the ongoing Dark Energy Survey (DES) will obtain deep griz imaging over a very large area that includes the entire SSDF down to approximately $24 \mathrm{mag}(\mathrm{AB} ; 10 \sigma)$.

\subsection{Spitzer/IRAC Data}

The SSDF, a post-cryogenic Spitzer Exploration Science program, obtained $120 \mathrm{~s}$ depth observations in the $3.6 \mu \mathrm{m}$ and $4.5 \mu \mathrm{m}$ IRAC bandpasses (hereafter, [3.6], [4.5]). Ashby et al. (2013) provide detailed information on the survey design, observations, processing, source extraction, and publicly available data products. Our study is based on the $4.5 \mu \mathrm{m}$ selected Spitzer/IRAC band-merged ([3.6], [4.5]) catalog, which contains $\sim 3.7$ million distinct sources down to the SSDF $5 \sigma$ sensitivity limit of $21.46 \mathrm{AB}$ mag $(9.4 \mu \mathrm{Jy})$ at $4.5 \mu \mathrm{m}$; the corresponding $5 \sigma$ sensitivity of the $3.6 \mu \mathrm{m}$ bandpass is $21.79 \mathrm{AB}$ mag (7.0 $\mu \mathrm{Jy})$. Throughout this paper, we use aperture-corrected, 4 " diameter aperture magnitudes for the IRAC data.

\subsection{SuperCOSMOS Optical Data}

The SuperCOSMOS survey (Hambly et al. 2001) provides allsky optical photometry based on scans of photographic Schmidt survey plates from the UK Schmidt Telescope (UKST) Southern Surveys (Hartley \& Dawe 1981; Cannon 1984) and Palomar Oschin Schmidt Telescope Surveys (POSS; Minkowski \& Abell 1963; Reid et al. 1991). These shallow data provide I-band magnitudes down to $I \sim 20.45$ mag (AB) in the SSDF (Hambly et al. 2001). As discussed above, portions of the SSDF have deeper optical data from the BCS and, eventually, the entire SSDF will be covered by the DES. However, in the interest of uniformity in our cluster search over the widest possible area, we only consider the relatively shallow SuperCOSMOS optical data in the following analysis.

\section{IDENTIFYING HIGH-REDSHIFT GALAXY CLUSTERS}

\subsection{Methodology}

We select candidate distant galaxy clusters based on their [3.6]-[4.5] galaxy color, following the approach of Papovich (2008); that methodology was proven effective by discovering a $z=1.62$ galaxy cluster in the SWIRE-XMM field (Lonsdale et al. 2003) using data of a depth similar to what is available in the SSDF (Papovich et al. 2010). The method takes advantage of the fact that the [3.6]-[4.5] color is a linear function of redshift between $0.7 \lesssim z \lesssim 1.5$ (Figure 1 ), and thus can be used as an effective redshift indicator. At $z \gtrsim 1.5$, the color reaches a plateau out to $z \sim 3$. The basis of the selection is that galaxy stellar populations with ages $>10$ Myr have a prominent bump at $\sim 1.6 \mu \mathrm{m}$ due to a minimum in the opacity of the $\mathrm{H}^{-}$ ion present in the atmospheres of cool stars. This feature is seen in the spectral energy distribution (SED) of essentially all galaxies, largely independent of star formation history or age (John 1988; Simpson \& Eisenhardt 1999; Sawicki 2002; Sorba $\&$ Sawicki 2010). However, above redshift $z \sim 1.5$ the color is not precise enough to be useful in estimating redshifts other than to constrain the redshift to be larger than $z \gtrsim 1.5$.

As shown in the middle panel of Figure 1, while an IRAC color cut [3.6]-[4.5] $>-0.1$ is effective at distinguishing galaxies at $z>1.3$, having at least one relatively shallow optical band is very useful for alleviating contamination from foreground interlopers at $z \sim 0.3$ (see discussion in Muzzin et al. 2013b). With this aim, we apply magnitude cuts in the $I$ and [4.5] bands to remove most $z<0.4$ galaxies (see bottom and top panel of Figure 1). Combined, these cuts effectively remove the bulk 


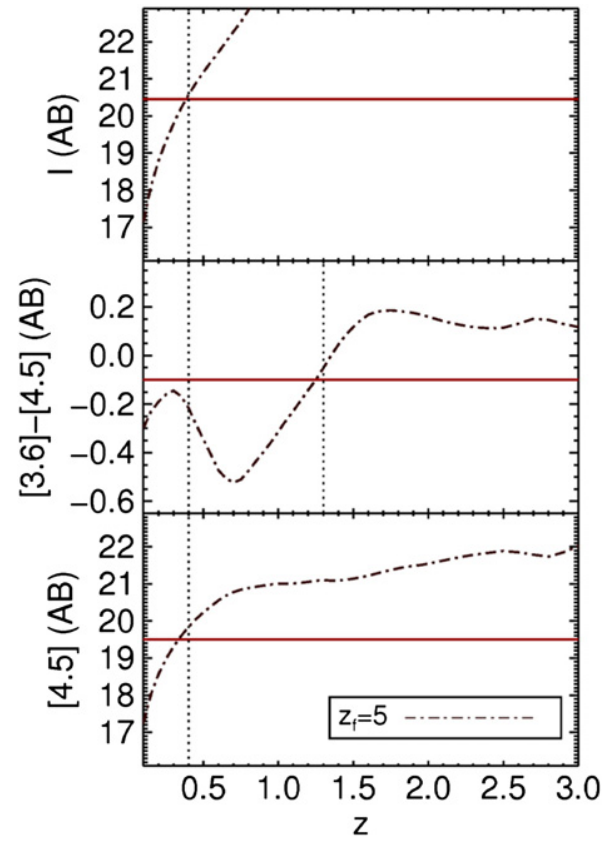

Figure 1. Evolution with redshift of SuperCOSMOS I-band magnitude (top panel), [3.6]-[4.5] IRAC color (middle panel), and [4.5] IRAC magnitude (bottom panel) for Bruzual \& Charlot (2003) simple stellar population models formed at formation redshifts, $z_{f}$, of 5 (black dot-dashed line). The IRAC color selection criterion we adopt is optimized to find galaxies with $z>1.3$. The [3.6]-[4.5] color serves as a good redshift indicator for $0.7 \lesssim z \lesssim 1.5$; above this redshift, the [3.6]-[4.5] color evolution with redshift flattens. To alleviate a known contamination from foreground interlopers at $z \sim 0.3$ (e.g., Papovich 2008; Muzzin et al. 2013b), we also apply two magnitude cuts ([4.5] > 19.5 and $I>20.45$ ).

(A color version of this figure is available in the online journal.)

of the foreground galaxy population at $z<1.3$. The remaining sources consist predominantly of high-redshift galaxies. We note that other sources of contamination are cool brown dwarfs (Stern et al. 2007) and powerful active galactic nuclei at all redshifts (Stern et al. 2005); however, these are expected not to be contaminants (see discussion in Galametz et al. 2012).

Therefore, to discover distant clusters of galaxies at $z>1.3$, we have implemented a simple three-filter algorithm to search for overdensities of galaxies based on their Spitzer IRAC color ([3.6]-[4.5] > -0.1), their 4.5 $\mu \mathrm{m}$ magnitude ([4.5] > 19.5), and requiring non-detection in the SuperCOSMOS $I$-band data $(I>20.45)$. Similar algorithms have been demonstrated to be effective using several programs (Papovich et al. 2010; Galametz et al. 2010, 2013; Gettings et al. 2012; Muzzin et al. 2013b).

To identify candidate galaxy clusters, for each galaxy in the SSDF catalog that meets the aforementioned criteria we count the number of similarly selected companions within $1^{\prime} .0$ radius cells. We correct these counts in cells for completeness using the values published in Table 5 of Ashby et al. (2013), who used a Monte Carlo approach to estimate the survey completeness and sensitivity by placing hundreds of simulated sources with randomly assigned Vega magnitudes (between 10 and 21 mag) in the SSDF mosaics at random locations (accounting realistically for the effects of source confusion). Ashby et al. (2013) then photometered the artificial sources in a manner identical to that used for the original mosaics. The process was iterated, so that a total population of 20,000 simulated sources was ultimately analyzed in each of the $0.5 \mathrm{mag}$ wide bins. This procedure was repeated in both bands. The results are given in their Table 5 and shown in their Figure 5. We also note that at the depths

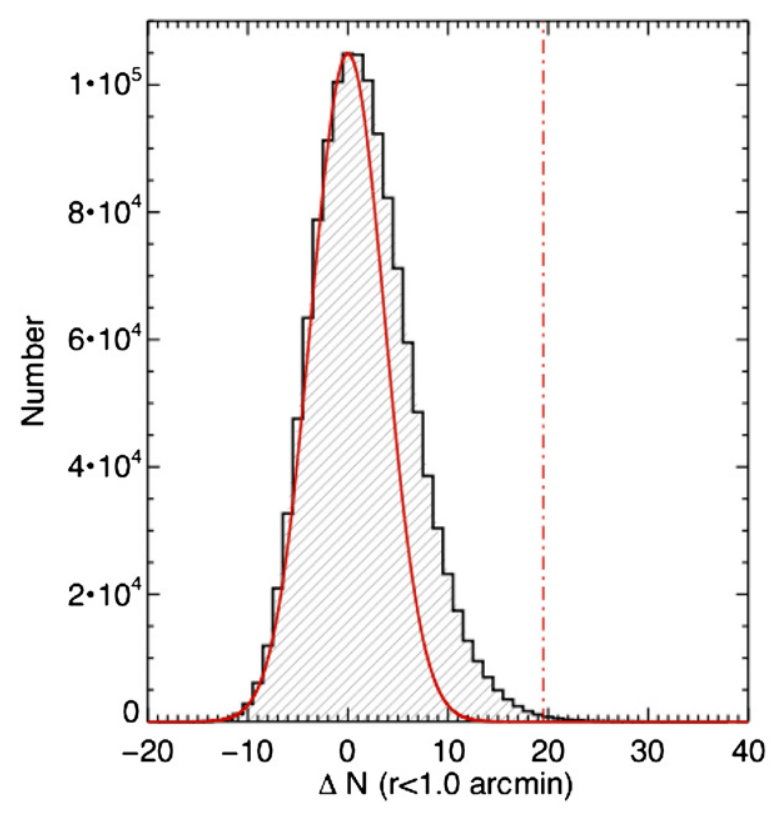

Figure 2. Distribution of the completeness-corrected excess number of objects with respect to the local background, $\Delta N$, with [3.6]-[4.5] $>-0.1,19.5<$ [4.5] $<21.46$, and $I>20.45$, within 1.0 radius from each individual source in the SSDF catalog fulfilling the aforementioned color criteria. The mean number of excess objects is $\langle\Delta N\rangle=0.4 \pm 3.7$. The red curve shows a Gaussian fit (iteratively clipping at $2 \sigma$ ). The dashed line indicates the minimum detection significance threshold adopted here of 5.2 times the standard deviations of the Gaussian distribution, corresponding to objects with more than 19.5 similarly selected (completeness-corrected) companions within 1'.0.

(A color version of this figure is available in the online journal.)

considered in our analysis, the completeness corrections vary, as a function of magnitude, between 1.3 and $11.5 \%$.

When creating an IRAC mosaic, the mapping strategy adopted results in some variations in depth across a large field. Therefore, we search for cells with the highest significance overdensity of IRAC-selected sources above the local background. With this aim, for each selected object in the catalog, we also count the completeness-corrected number of similarly selected companions in an outer annulus defined by radii of $5^{\prime}$ and $8^{\prime}$. After taking into account the difference in surface areas, we then derive the excess number of selected companions with respect to the local background, $\Delta N$.

Figure 2 shows the distribution of the completeness-corrected excess number of companions, $\Delta N$, within $1{ }^{\prime} .0$ radius. The mean number of excess galaxies is $\langle\Delta N\rangle=0.4$. The distribution is skewed toward objects with higher-than-average numbers of companions, suggestive of strong clustering. Similar to Papovich (2008), we fit a Gaussian to the distribution, iteratively clipping at $2 \sigma$. The best fit is shown in Figure 2 and it matches well with the low-excess half of the distribution. We find the width of the fitted Gaussian to be $\sigma_{\Delta N}=3.7$.

The minimum detection significance to adopt, $X_{f_{\min }}$, is somewhat arbitrary, trading between completeness and reliability in the derived candidate cluster catalog. We based our conservative choice on extensive tests performed on similar-depth IRAC and SuperCOSMOS data in the Boötes field, where ancillary spectroscopic and much deeper multi-wavelength photometric coverage is also present. We describe these tests in Section 3.2, where we estimate that the threshold adopted here ensures a purity of $\sim 80 \%$. Thus, we define our sample of candidate clusters of galaxies to be those with $\Delta N \geqslant\langle\Delta N\rangle+X_{f_{\min }} \cdot \sigma_{\Delta N}$, with $X_{f_{\min }}=5.2$. For the SSDF field, this corresponds to objects 
Table 1

Top 10 Most Significant SSDF $z>1.3$ Galaxy Cluster Candidates

\begin{tabular}{lccc}
\hline \hline SSDF ID & $\begin{array}{c}\text { R.A. } \\
(\text { deg., J2000) }\end{array}$ & $\begin{array}{c}\text { Dec. } \\
(\text { deg., J2000) }\end{array}$ & $\begin{array}{c}\text { Significance } \\
\left(X_{f}\right)\end{array}$ \\
\hline SSDF-CLJ2339-5531 & 354.786 & -55.5308 & 9.10 \\
SSDF-CLJ0000-5540 & 0.04973 & -55.6782 & 8.77 \\
SSDF-CLJ2340-5403 & 355.235 & -54.0649 & 8.39 \\
SSDF-CLJ2316-5317 & 349.072 & -53.2995 & 7.98 \\
SSDF-CLJ2357-5800 & 359.440 & -58.0069 & 7.82 \\
SSDF-CLJ2330-5037 & 352.601 & -50.6244 & 7.69 \\
SSDF-CLJ2312-5253 & 348.113 & -52.8975 & 7.56 \\
SSDF-CLJ2322-5156 & 350.531 & -51.9481 & 7.49 \\
SSDF-CLJ2317-5642 & 349.301 & -56.7126 & 7.38 \\
SSDF-CLJ2334-5053 & 353.632 & -50.8836 & 7.38 \\
\hline
\end{tabular}

with $\Delta N \geqslant 19.5$ excess companions with respect to the local background (indicated by the vertical dot-dashed red line of Figure 2).

By design, many of the objects in overdense regions will be identified as companions to multiple sources by our algorithm. To address this, we adopt an approach similar to that in Papovich (2008). We merge cluster candidates by applying a friends-offriends algorithm with a linking length of 1'.0. This results in a final catalog containing $N_{\text {obs }}=279$ candidate clusters; their spatial distribution and color-coded detection significance are shown in Figure 3. We note that none of our clusters is found in the list of SPT SZ-detected clusters reported in Reichardt et al. (2013). This is not unexpected, since the two samples do not overlap in mass-redshift space: the highest-redshift cluster in Reichardt et al. (2013) is at $z=1.075$, below the range to which our cluster-finding algorithm is sensitive, while the typical mass of the clusters we find is below the mass threshold of the Reichardt et al. (2013) catalog.

In Table 1, we present the list of the top 10 most significant cluster candidates in the SSDF field. Figures 4 and 5 present their images and color-magnitude diagrams. The coordinates listed in Table 1 refer to the galaxy at the center of the cell containing the highest number of cluster member candidates.

\subsection{Purity of the SSDF Cluster Candidates}

For a large sample of candidate clusters to be useful for galaxy evolution and cosmological studies, it is important to determine its purity $f_{\text {pure }}$ as a function of the detection significance $X_{f}$, defined as

$$
f_{\text {pure }}\left(X_{f}\right)=\frac{N_{\text {real }}}{N_{\text {tot }}}=1-\frac{N_{\text {false }}}{N_{\text {tot }}},
$$

where $N_{\text {tot }}$ is the total number of cluster candidates above the detection threshold $X_{f}, N_{\text {real }}$ is the number of candidates corresponding to real clusters, and $N_{\text {false }}$ is the number of false detections. With this aim, we run our cluster-finding algorithm on comparable-depth observations from the IRAC Shallow Cluster Survey (ISCS; Eisenhardt et al. 2004).

The ISCS is a wide-field IR-selected galaxy cluster survey carried out using 90 s Spitzer/IRAC imaging of the $8.5 \mathrm{deg}^{2}$ Boötes field of the NOAO Deep, Wide-Field Survey (NDWFS; Jannuzi \& Dey 1999). SuperCOSMOS data are available in Boötes of a depth comparable to the data available in the SSDF field. Over the last decade, the Boötes cluster candidates have been the target of extensive ground- and space-based spectroscopic and photometric campaigns (e.g., Eisenhardt et al. 2008; Ashby et al. 2009; Stern et al. 2010; Brodwin et al. 2011;
Stanford et al. 2012; Zeimann et al. 2012; Gonzalez et al. 2012; Brodwin et al. 2013). Using a wavelet search algorithm operating on photometric-redshift probability distribution functions, as described in Brodwin et al. (2006), more than 100 rich cluster candidates at $z>1$ were identified. To date, 18 of these have been spectroscopically confirmed out to $z=1.9$. Note that very accurate photometric redshift measurements are based on data from the Spitzer Deep, Wide-Field Survey (SDWFS; Ashby et al. 2009, 4× deeper than the IRAC data used here), deep optical imaging in $B_{W}, R, I$ bands from the NDWFS, and NIR photometry from the FLAMEX survey (Elston et al. 2006) in the $J$ and $K_{S}$ bands. Note, however, that the ISCS is ongoing, with many cluster candidates still awaiting confirmation. In the absence of a complete, spectroscopically confirmed catalog of high-redshift clusters, we deem the ISCS the best available survey to test our algorithm, as it contains the largest sample $(>10)$ of spectroscopically confirmed clusters at $z>1.3$.

We find the purity of our sample, $f_{\text {pure }}$, to be a monotonic function of $X_{f}$ reaching $f_{\text {pure }}=0.8$ for $X_{f_{\min }}=5.2$. At this very high detection significance, our algorithm identifies 14 candidate clusters at $z>1.3$ in the Boötes field, of which 3 are spectroscopically confirmed at redshifts of $z=1.37$ (Brodwin et al. 2013; Zeimann et al. 2013), $z=1.41$ (Stanford et al. 2005; Brodwin et al. 2011), and $z=1.75$ (Stanford et al. 2012), and an additional eight have accurate photometric redshifts $1.3<z_{\text {phot }}<2.3$. In the left panel of Figure 6 , we show the [3.6]-[4.5] color versus [3.6] magnitude for the cluster IDCS $\mathrm{J} 1426.5+3508$ at $z=1.75$ as found by our algorithm with $X_{f}=5.3$. This cluster, spectroscopically confirmed with $H S T$ / WFC3 grism observations (Stanford et al. 2012), was detected in both archival $8.3 \mathrm{ks}$ Chandra imaging of the field (Stanford et al. 2012) as well as follow-up SZ observations with the CARMA array (Brodwin et al. 2012). The cluster also has a giant arc in HST imaging, implying it is a lensing cluster; this is particularly surprising given the cluster redshift and the small area of sky surveyed (Gonzalez et al. 2012). At the time of its discovery, IDCS J1426.5+3508 was the highest redshift cluster for which the SZ effect had been measured. One of the other confirmed clusters, ISCS J1438.1+3414 at $z=1.41$, was the most distant cluster known at the time of its discovery (Stanford et al. 2005). Follow-up Chandra observations detected the cluster in the X-ray (Andreon et al. 2011; Brodwin et al. 2011). This cluster is found by our algorithm with the highest significance ( $X_{f}=8.64$; see the middle panel of Figure 6 ) in the Boötes field. The only other confirmed high-redshift Boötes cluster with $\mathrm{X}$-ray emission is ISCS J1432.4+3250 at $z=1.49$, which is also identified as an overdense region by our algorithm, though at a level slightly below the very conservative threshold adopted here $\left(X_{f}=4.2\right.$; see the right panel of Figure 6). The red-sequence color and scatter for the known spectroscopically confirmed clusters in Boötes is very similar to the one shown by the SSDF candidates throughout the entire range of detection significance, down to $X_{f_{\min }}=5.2$ (as illustrated in Figure 7). To summarize, the conservative cut in detection significance we have derived from this analysis yields many previously identified clusters in the Boötes survey out to $z=1.8$, lending confidence in the effectiveness of our cluster-finding algorithm when applied to similar-depth data in the SSDF.

\section{CLUSTERING OF HIGH-REDSHIFT CLUSTERS}

Clusters of galaxies reside in the largest dark matter halos, representing higher density peaks of the mass distribution as the redshift increases (Springel et al. 2005). Thus, the number 

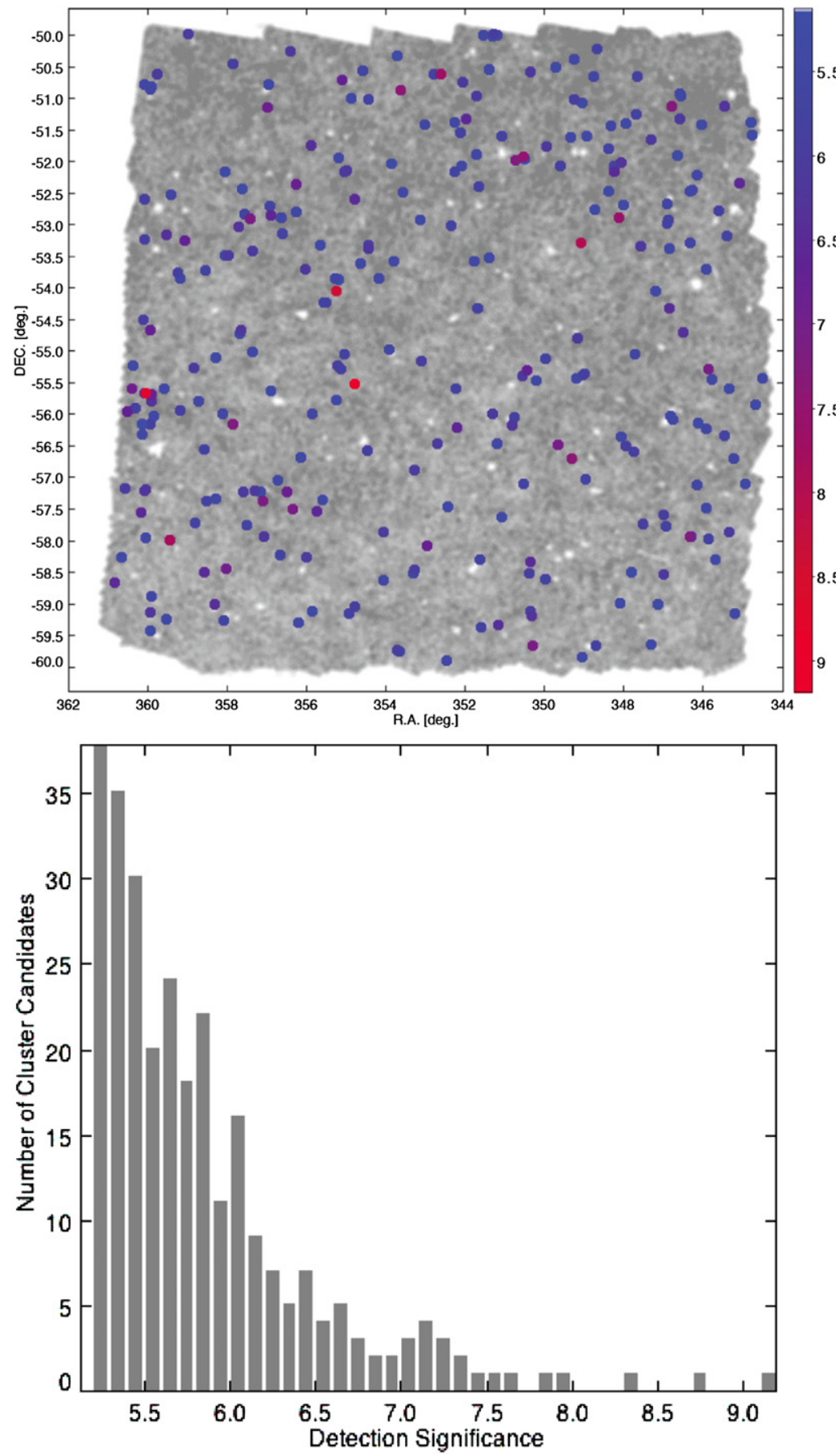

Figure 3. Top panel: spatial distribution of 279 galaxy cluster candidates with $X_{f} \geqslant 5.2$ detection significance in the SSDF. Each data point corresponds to one cluster candidate defined in Section 3.1, color-coded by detection significance. The gray background shows the positions of all galaxies in the SSDF field that meet the color criteria described in the text. Note that the white gaps in the background map are due to masked areas surrounding bright stars. Bottom panel: distribution of the detection significance, $X_{f}$, for the 279 galaxy cluster candidates.

(A color version of this figure is available in the online journal.)

density of galaxy clusters with redshift is sensitive to the cosmic matter density, $\Omega_{\mathrm{m}}$, and its evolution with redshift provides constraints on cosmological parameters (e.g., Kitayama \& Suto 1996; Wang \& Steinhardt 1998).

Several observational studies have previously demonstrated that cluster samples show strong clustering (e.g., Bahcall 1988; Huchra et al. 1990; Postman et al. 1992; Borgani et al. 1999; Gonzalez et al. 2002; Bahcall et al. 2003; Brodwin et al. 2007; Papovich 2008) as measured by their autocorrelation function (Peebles 1980).
High-resolution simulations predict that the cluster correlation function strength increases with redshift for a given mass limit. That is, high-redshift clusters are more strongly clustered, on a comoving scale, than low-redshift clusters of the same mass (Bahcall et al. 2003). Moreover, a relative constancy of the cluster correlation function strength for the most massive clusters at every epoch is predicted: the $N$ most massive clusters at one epoch should have clustering similar to the $N$ most massive clusters at a later epoch (Younger et al. 2005). 

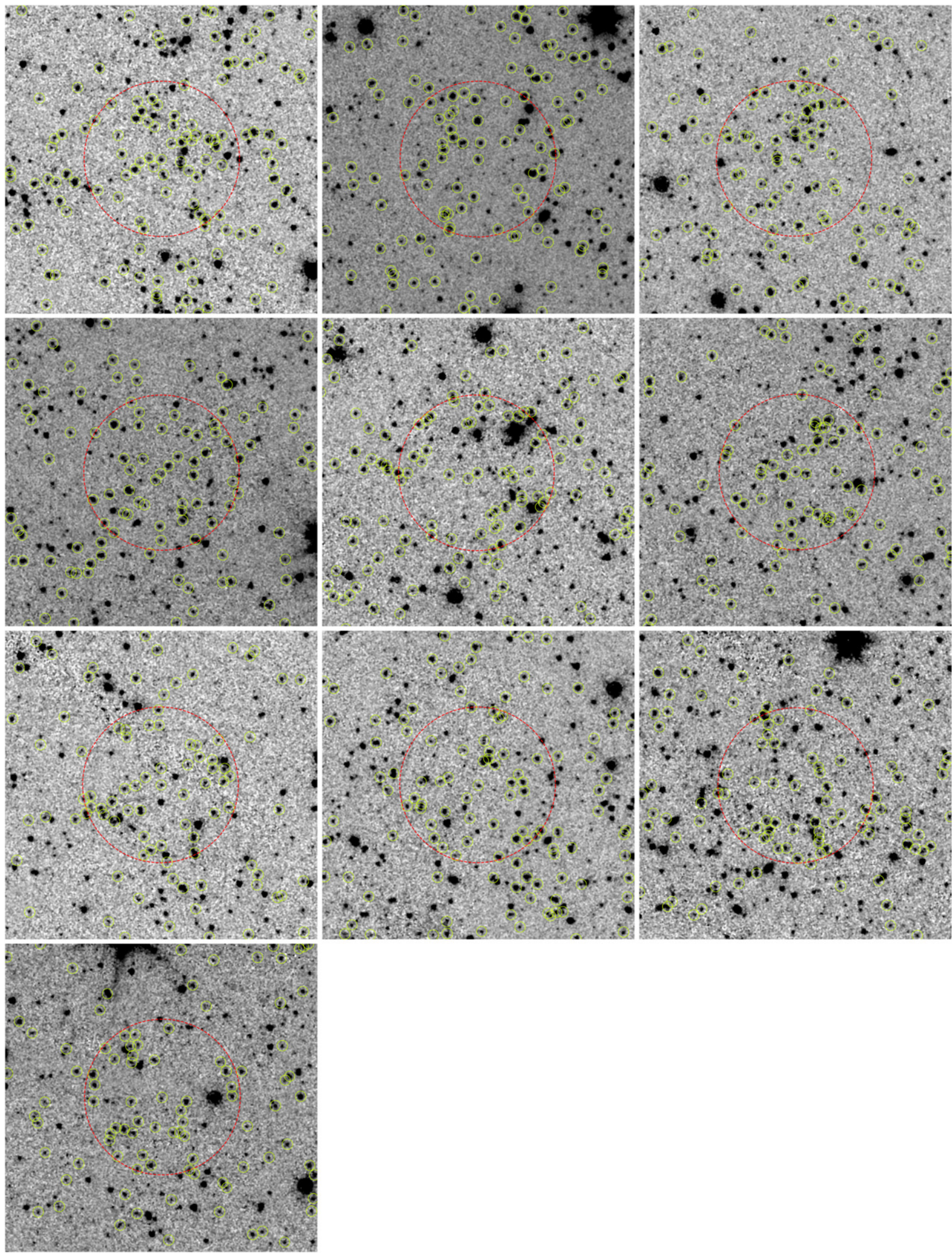

Figure 4. Spitzer/IRAC $4.5 \mu \mathrm{m}$ images $\left(4^{\prime} \times 4^{\prime}\right)$ of the 10 most significant high-redshift candidate galaxy clusters in the SSDF survey. The yellow circles indicate candidate cluster members. The red circle has a radius of $1^{\prime} .0$, corresponding to an angular diameter distance of $\sim 0.5 \mathrm{Mpc}$ at $z=1.5$.

(A color version of this figure is available in the online journal.) 

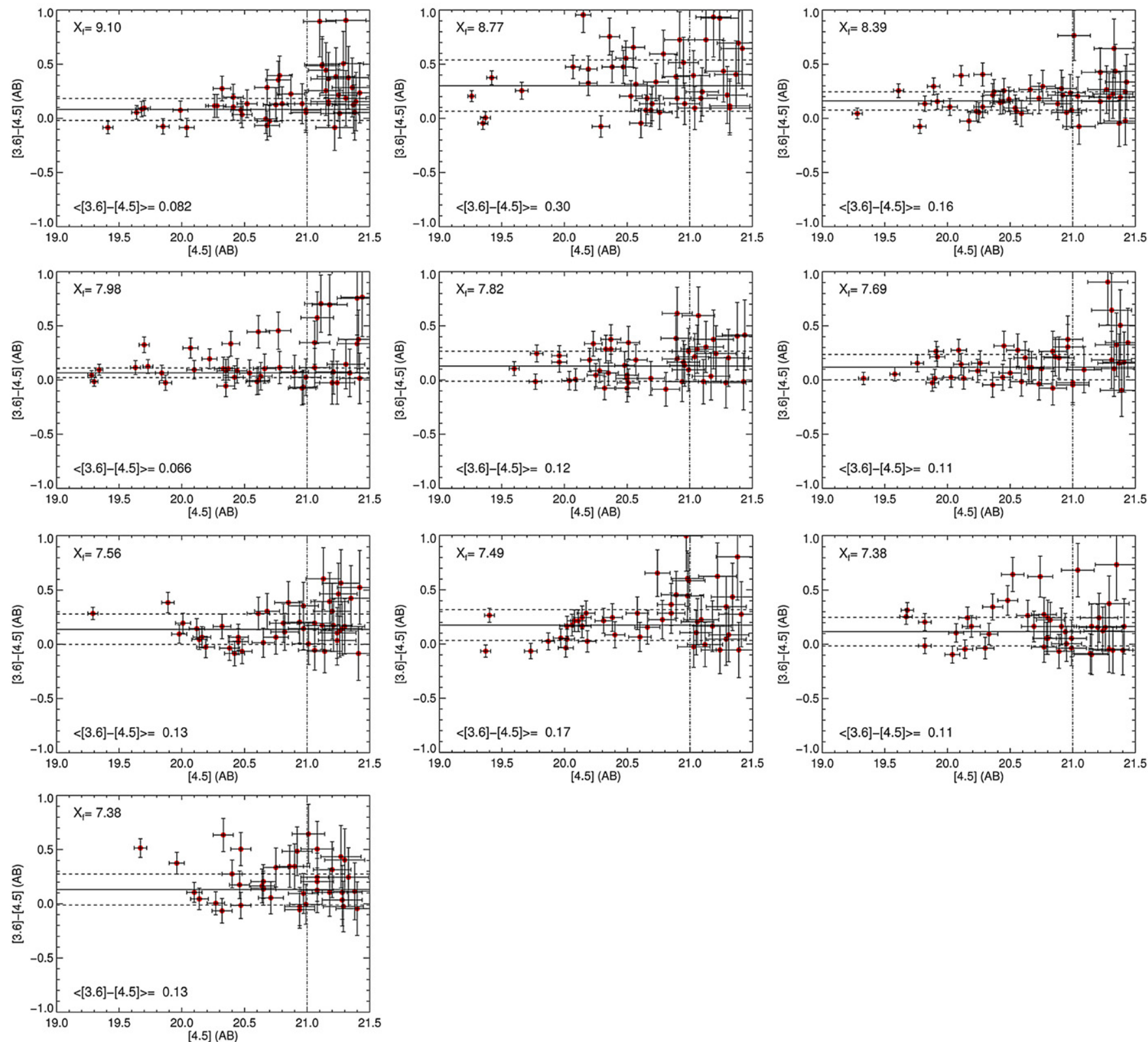

Figure 5. [3.6]-[4.5] color vs. [4.5] magnitude for the 10 most significant high-redshift candidate galaxy clusters in the SSDF survey. The solid lines indicate the median [3.6]-[4.5] color for member galaxies with [4.5] $<21$. The dashed lines indicate $\pm \sigma$, the standard deviation of the color distribution.

(A color version of this figure is available in the online journal.)
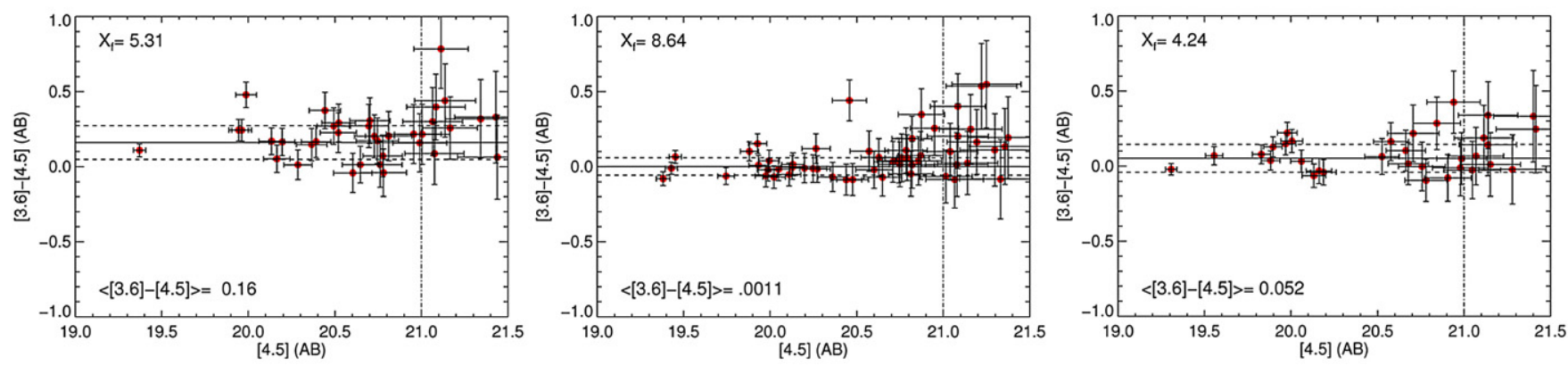

Figure 6. [3.6]-[4.5] color vs. [4.5] magnitude for the IDCS J1426.5+3508 cluster confirmed at $z=1.75$ (left panel), as found by our algorithm with one of the highest detection significances $\left(X_{f}=5.31\right)$ when applied to comparable-depth observations from the ISCS. Color-magnitude diagrams are also shown for ISCS $\mathrm{J} 1438.1+3414$ at $z=1.41\left(X_{f}=8.64\right.$, middle panel) and ISCS J1432.4+3250 at $z=1.49\left(X_{f}=4.24\right.$, right panel $)$, the two confirmed ISCS clusters with X-ray emission also found by our algorithm at high $X_{f}$. Lines and symbols are similar to those adopted for Figure 5 .

(A color version of this figure is available in the online journal.) 

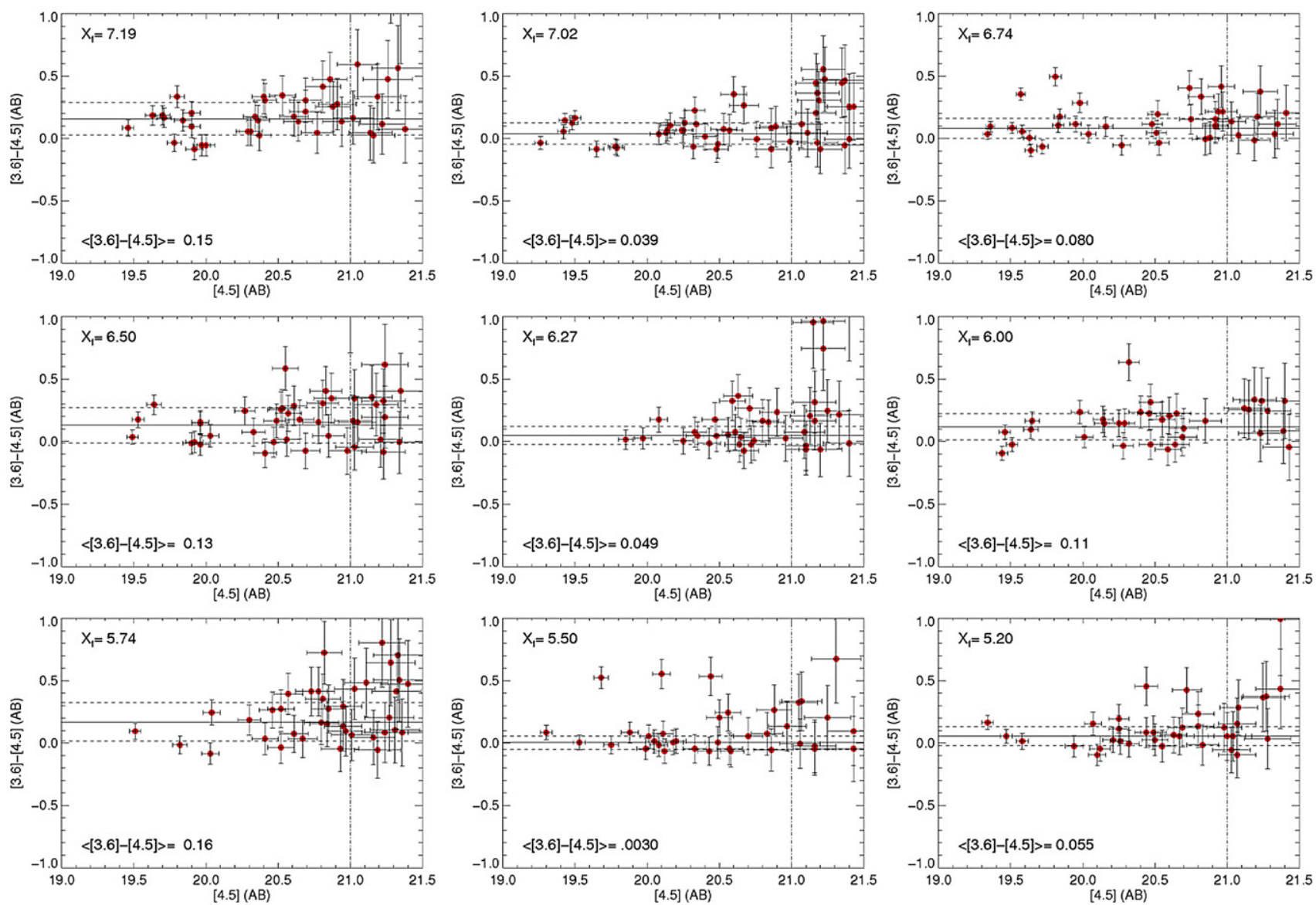

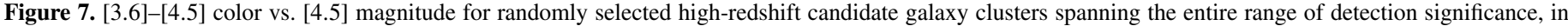
bins of $\Delta X_{f}=0.25$, down to $X_{f_{\min }}=5.2$. Lines and symbols are similar to those adopted for Figure 5 .

(A color version of this figure is available in the online journal.)

Currently, both the measured correlation function scale lengths and number densities of several galaxy cluster samples support predictions from standard cold dark matter models ( $\Lambda$ CDM; e.g., Abadi et al. 1998; Croft et al. 1997; Peacock \& West 1992; Bahcall et al. 1993; Collins et al. 2000; Bahcall et al. 2003; Brodwin et al. 2007; Papovich 2008; Vikhlinin et al. 2009; Hasselfield et al. 2013). Next, we measure these quantities for our cluster sample in order to check our consistency with previous observational studies and model predictions.

\subsection{Comoving Number Density}

In order to derive the comoving number density of our cluster sample, we first need to infer the expected redshift distribution $\phi(z)$ of our sample. With this aim, we employ the COSMOS/ UltraVista photometric redshift catalog from Muzzin et al. (2013a). This catalog includes $3.6 \mu \mathrm{m}$ and $4.5 \mu \mathrm{m}$ photometry, reaching 2 magnitudes deeper than the SSDF. The central panel in Figure 8 shows the smoothed [3.6]-[4.5] color versus redshift distribution of the COSMOS catalog; details on this filtering procedure can be found in Martinez-Manso et al. (2015). The white line marks the [3.6]-[4.5] $>-0.1$ selection, and the magenta curve follows the peak of the full galaxy distribution. We parameterize this curve with redshift and denote it as $\mathcal{S}(z)$. At a given redshift, we expect the color distributions of cluster and field galaxies to have the same centroid (given by $\mathcal{S}(z)$ ), but with a smaller scatter for the clusters given their more homogeneous star formation histories and faster evolution (Rettura et al. 2010). As pointed out in Section 3.1, a significant contamination by
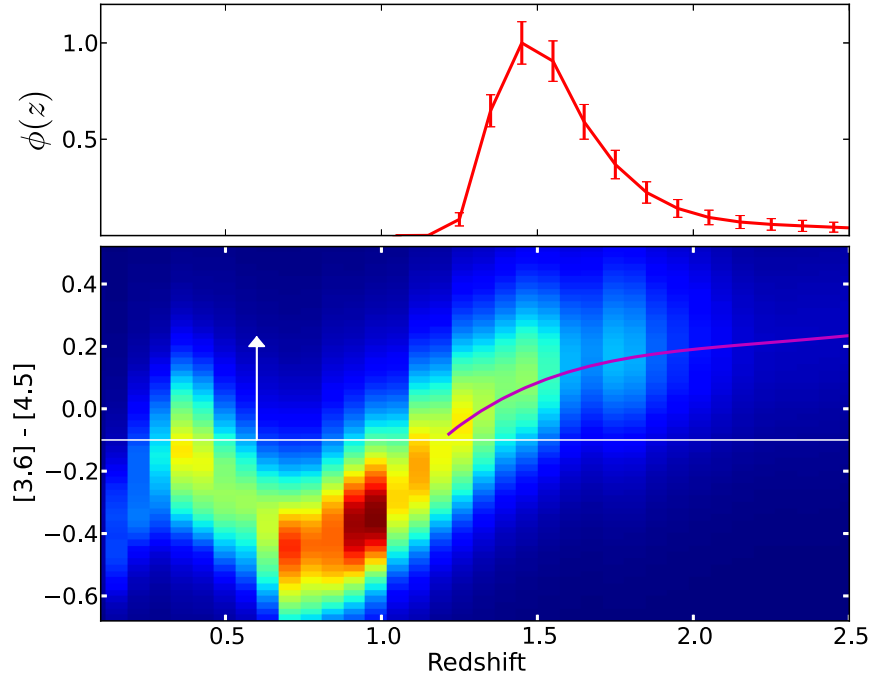

Figure 8. Bottom panel: [3.6]-[4.5] color vs. redshift distribution of the COSMOS/UltraVista galaxy catalog. In the SSDF, we select galaxies with [3.6]-[4.5] > -0.1, marked as the horizontal white line. The magenta curve follows the peak of the distribution, where cluster colors will most likely be centered. Top panel: redshift distribution of our cluster sample.

(A color version of this figure is available in the online journal.)

$z \sim 0.3$ occurs with a simple IRAC color cut. As we remove this contamination with optical and [4.5] magnitude cuts, in the following analysis we assume that all our clusters are at $z>1$. 
To analyze the colors of our cluster candidates, we consider the 10 brightest galaxies in [4.5] from each of them (the total number of member candidates is $\sim 40$ per cluster). The goal of this selection is twofold: it removes faint galaxies, which are more likely to be contaminants due to photometric errors, and keeps the most massive galaxies, which are more likely to share a similar star formation history. The typical scatter in color in clusters after applying this selection is $\sim 0.12 \mathrm{mag}$. In comparison, field galaxies at a given redshift have a much larger color scatter of $\sim 0.25 \mathrm{mag}$, confirming our expectation that the cluster members are a more homogeneous population.

At this point, we have established that our clusters are likely to follow the redshift-color relation given by $\mathcal{S}(z)$. In order to derive the cluster redshift distribution and its errors, we apply a bootstrap procedure on the cluster colors. In each iteration, a new color for each cluster is drawn from a normal distribution, with the center and standard deviation equal to the mean and standard error of the colors of the 10 brightest member candidates, respectively. These cluster colors are mapped to redshift using $\mathcal{S}(z)$ and binned onto a fixed redshift grid. After 100 iterations, we compute the mean and standard deviation of all values in each redshift bin, yielding the cluster redshift distribution shown in the top panel of Figure 8.

We can calculate the spatial number density of the cluster sample at the pivot redshift $z_{p} \equiv 1.5$ by combining the redshift distribution $\phi(z)$, the SSDF survey area, and the number of cluster candidates, $N_{\text {obs }}$. The number of clusters within $z_{p} \pm \delta z / 2$ can be written as

$$
\Delta N=N_{\mathrm{obs}} \frac{\phi\left(z_{p}\right)}{\int \phi\left(z^{\prime}\right) d z^{\prime}} \delta z .
$$

The sampled volume is

$$
\Delta V=\frac{d V\left(z_{p}\right)}{d z} \delta z=\frac{c \Omega \chi^{2}\left(z_{p}\right)}{H\left(z_{p}\right)} \delta z
$$

where $\chi(z)$ is the comoving radial distance, $H(z)$ is the Hubble function, $c$ is the speed of light, and $\Omega=0.0271 \mathrm{sr}$ is the solid angle subtended by the SSDF survey. Hence, the number density for our SSDF cluster sample, $n_{\mathrm{c}}$, at $z=1.5$ is

$$
\begin{aligned}
n_{\mathrm{c}}=\frac{\Delta N}{\Delta V}= & (7.6 \pm 0.6) \times 10^{-7} h^{3} \mathrm{Mpc}^{-3} . \\
& \text { 4.2. Halo Model }
\end{aligned}
$$

\subsection{Halo Model}

Our infrared selection of galaxies and the ranking system we adopt for our cluster search algorithm is expected to produce a nearly mass-limited cluster sample at $z>1.3$. Thus, we assume that our cluster sample is comprised of mostly dark matter halos above some characteristic minimum mass $M_{\min }$, following the halo occupation framework (Ma \& Fry 2000; Seljak 2000; Peacock \& Smith 2000; Scoccimarro et al. 2001; Cooray \& Sheth 2002; Berlind \& Weinberg 2002; Berlind et al. 2003; Kravtsov et al. 2004; Zheng et al. 2005). We model the probability of a halo of mass $M$ to be part of the cluster sample as

$$
N_{c}(M)=\left\{\begin{array}{ll}
1 & \text { if } M \geqslant M_{\min } \\
0 & \text { if } M<M_{\min }
\end{array}\right\} .
$$

Then, the comoving number density of clusters is determined to be

$$
n_{c}=\int_{M_{\mathrm{low}}}^{M_{\mathrm{high}}} d M \frac{d n}{d M}(M) N_{c}\left(M \mid M_{\mathrm{min}}\right),
$$

where $(d n / d M)(M)$ is the halo mass function from Tinker et al. (2010) and the integration limits are $M_{\text {low }}=10^{11} M_{\odot}$ and $M_{\text {high }}=10^{16} M_{\odot}$. In addition, we can calculate the mean mass of the cluster sample as an average over the occupied halos:

$$
\begin{gathered}
M_{\text {mean }}=\frac{1}{n_{c}} \int d M \frac{d n(M)}{d M} N_{c}\left(M \mid M_{\min }\right) M . \\
\text { 4.3. Angular Correlation Function }
\end{gathered}
$$

An additional observable that links the distribution of dark matter halos and our clusters is the measurement of their clustering. In particular, we focus on the modeling of the twopoint spatial correlation function (SCF, formally $\xi(r)$; Peebles 1980), which represents the excess probability of finding two objects at a separation $r$ with respect to the case of a randomly distributed sample. The formation of dark matter halos of a given mass $M$ is directly correlated with the dark matter overdensity at their location (Kaiser 1984; Fry \& Gaztanaga 1993; Mo \& White 1996). This translates into a scaling between the dark matter and halo SCFs, which is called the halo bias $b_{h}$ :

$$
\xi_{h}(M, r, z)=\xi_{d m}(r, z) b_{h}^{2}(M, z)
$$

Our definition of halo mass is that enclosed by a sphere with a density 200 times larger than the critical density of the universe. We obtain the dark matter correlation function from the CAMB software (Lewis \& Bridle 2002) and the halo bias from Sheth et al. (2001), using the updated parameters from Tinker et al. (2005). The bias of the clusters with respect to dark matter is then

$$
b_{c}=\frac{1}{n_{c}} \int d M \frac{d n(M)}{d M} N_{c}\left(M \mid M_{\min }\right) b_{h}(M) .
$$

Since the observed configuration space of our cluster sample is the celestial sphere, we measure its clustering via the angular correlation function (ACF, formally $\omega(\theta))$. The ACF can be considered the radial projection of the SCF, which can be computed with the knowledge of the sample's redshift distribution (Limber 1953; Phillipps et al. 1978):

$$
\omega(\theta)=\frac{2}{c} \int_{0}^{\infty} d z H(z) \phi^{2}(z) \int_{0}^{\infty} d y \xi_{c}\left(r=\sqrt{y^{2}+D_{c}^{2}(z) \theta^{2}}\right)
$$

where $\phi(z)$ is the normalized redshift distribution, $D_{c}(z)$ is the radial comoving distance, $c$ is the speed of light, and $\theta$ is the angular separation given in radians. We measure $\omega(\theta)$ with the estimator presented in Hamilton (1993), which counts the number of galaxy pairs with respect to those of a random sample distributed in the same geometry:

$$
\hat{\omega}(\theta)=\frac{\operatorname{RR}(\theta) \mathrm{GG}(\theta)}{(\mathrm{GR})^{2}(\theta)}-1,
$$

where GG, GR, and RR are total number of galaxy-galaxy, galaxy-random and random-random pairs separated by an angle $\theta$. There is no need to include a correction for the integral constraint (Peebles 1980), since it was shown in MartinezManso et al. (2015) that it is negligible for this very wide field survey geometry. We estimate $\omega(\theta)$ errors using jackknife resampling. For this, the entire sample is divided into $N_{\text {jack }}=32$ spatial regions of equal size. Then, the correlation is run $N_{\text {jack }}$ times, each time excluding one of those regions from the sample. The value of the estimator is the average $\bar{\omega}(\theta)$ of those iterations 


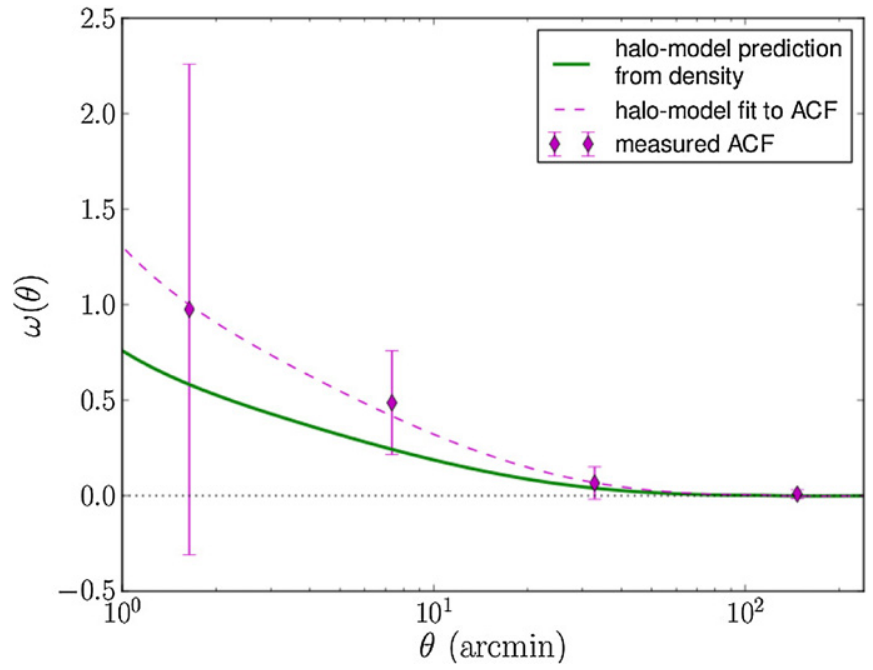

Figure 9. Observed clustering measured via the ACF (points), fitted $\omega(\theta)$ (dashed curve), and predicted $\omega(\theta)$ based on the observed number density (solid curve).

(A color version of this figure is available in the online journal.)

and the covariance between angular bins is given by

$$
C_{j k}=\frac{N-1}{N} \sum_{i=0}^{N}\left[\hat{\omega}_{i}\left(\theta_{j}\right)-\bar{\omega}\left(\theta_{j}\right)\right]\left[\hat{\omega}_{i}\left(\theta_{k}\right)-\bar{\omega}\left(\theta_{k}\right)\right] .
$$

The observed $\omega(\theta)$ function is shown in Figure 9, where the error bars are derived from the diagonal elements of the covariance matrix.

\subsubsection{Results}

The halo model of the clustering described so far can be fully specified either by fixing $n_{c}$ through the observed cluster counts (Equation (4)), or by fixing $b_{c}$ through the amplitude of the observed clustering. We refer to these two approaches as the density and clustering fits, respectively. Thus, comparing these sets of results is an excellent way to test the consistency of the model and observations. Our fits are based on the maximization of the likelihood of the model given the data, $\mathcal{L}(\bmod \mid$ data $)=e^{-\chi^{2} / 2}$. For the clustering fit, this is specified by

$$
\chi^{2}=\sum_{i=0}^{K} \sum_{j=0}^{K}\left[\omega_{m}\left(\theta_{j}\right)-\bar{\omega}\left(\theta_{j}\right)\right] C_{i j}^{-1}\left[\omega_{m}\left(\theta_{k}\right)-\bar{\omega}\left(\theta_{k}\right)\right] .
$$

Here, $\omega_{m}$ and $\bar{\omega}$ are the predicted and observed ACFs (Equations (10) and (11), respectively), $C_{i j}$ is the covariance matrix from Equation (12), and $K=4$ is the number of angular bins.

For the density fit,

$$
\chi^{2}=\left(n_{c}^{\text {mod }}-n_{c}^{\text {data }}\right)^{2} / \sigma_{n_{c}}^{2},
$$

where the variance $\sigma_{n_{c}}^{2}$ is derived from Poisson statistics in the cluster counts. We have neglected the errors from $\phi(z)$ after checking that their contribution to the final error budget is almost an order of magnitude smaller than the other sources being accounted for in either fit. Results are shown in Table 2. We have included the calculation of $r_{0}$, which marks the distance scale length where $\xi_{c}\left(r_{0}\right)=1$, with $\xi_{c}=\xi_{d m}\left(z=z_{p}\right) b_{c}^{2}$ for both methods.
Table 2

Best-fit Results for the Number Density, the Characteristic Minumum Mass, the Mean Mass, the Bias, and the Angular Correlation Length of the SSDF Cluster Sample as Found by the Density and the Clustering Fits

\begin{tabular}{lcc}
\hline \hline & Density & Clustering \\
\hline$n_{c}\left(10^{-7} h^{3} \mathrm{Mpc}^{-3}\right)$ & $7.6 \pm 0.6$ & $0.7_{-0.6}^{+6.3}$ \\
$M_{\min }\left(10^{14} h^{-1} M_{\odot}\right)$ & $0.78 \pm 0.02$ & $1.5_{-0.7}^{+0.9}$ \\
\hline$M_{\text {mean }}\left(10^{14} h^{-1} M_{\odot}\right)$ & $1.08 \pm 0.02$ & $1.9_{-0.8}^{+1.0}$ \\
\hline$b_{c}$ & $8.2 \pm 0.1$ & $10.8 \pm 2.5$ \\
\hline$r_{0}\left(h^{-1} \mathrm{Mpc}\right)$ & $25.9 \pm 0.4$ & $32 \pm 7$ \\
\hline
\end{tabular}

Overall, there is a reasonable agreement between the density and clustering sets, differing by less than $2 \sigma$. Throughout the paper, we make the conservative choice of adopting the clustering sets as fiducial values since they have the larger errors. The two angular correlation functions, $\omega(\theta)$, one corresponding to the prediction based on the number density (solid curve) and one based on the fit of the measured ACF points (dashed curve), are displayed in Figure 9 and found to be consistent within the errors.

The values of $n_{\mathrm{c}}$ and $r_{0}$ found for our sample of candidate clusters are consistent with those found by previous observational studies in the literature (Abadi et al. 1998; Collins et al. 2000; Bahcall et al. 2003; Brodwin et al. 2007, see also Figure 9 of Papovich 2008) and are well in agreement with predictions based on $\Lambda \mathrm{CDM}$ cosmological models (Springel et al. 2005). This analysis lends further confidence in the effectiveness of our cluster-finding algorithm.

\section{SUMMARY}

We have identified a large sample of massive high-redshift galaxy cluster candidates at $z>1.3$ over the $94 \mathrm{deg}^{2}$ Spitzer survey of the SPTpol field. Our algorithm identifies the most significant overdensities of galaxies based on their IRAC color ([3.6]-[4.5] > -0.1), their 4.5 $\mu \mathrm{m}$ magnitude ([4.5] > 19.5), and requiring non-detection in the shallow SuperCOSMOS $I$-band data $(I>20.45)$. We identify 279 distant cluster candidates using a $X_{f} \geqslant 5.2$ detection significance, for which we estimate an $\sim 80 \%$ purity by running our algorithm on comparable-depth observations of the Spitzer surveys of the Boötes field (Eisenhardt et al. 2004; Ashby et al. 2009), which has been the target of extensive ground- and space-based spectroscopic and photometric campaigns over the past decade.

We find that the SSDF cluster sample shows strong clustering. From the angular correlation analysis, we find that our sample has a comoving number density $n_{c}=\left(0.7_{-0.6}^{+6.3}\right) \times 10^{-7} h^{3} \mathrm{Mpc}^{-3}$ and a spatial clustering correlation scale length $r_{0}=(32 \pm$ 7) $h^{-1} \mathrm{Mpc}$. These values are consistent with previous observational studies and match expectations based on $\Lambda C D M$ highresolution simulations. The high-redshift cluster sample presented here has a mean mass $M_{\text {mean }}=1.9_{-0.8}^{+1.0} \times 10^{14} h^{-1} M_{\odot}$. Assuming these clusters grow according to predictions of $\Lambda$ CDM (e.g., Fakhouri et al. 2010), they will evolve into massive clusters $\left(>5 \times 10^{14} h^{-1} M_{\odot}\right)$ at $z=0.2$.

This study showcases the impact that large Warm Spitzer surveys can have on the identification of large samples of massive clusters of galaxies at very high redshifts in the upcoming years. In particular, this sample has been selected in an area where deep observations for the SZ effect with the SPTpol camera are underway, and part of this field also has XMM-Newton deep X-ray observations from the XXL Survey. 
These ancillary data will allow us to determine cluster masses for our sample, enabling a systematic study of the cluster population in a crucial epoch for their assembly.

A.R. is grateful to the SSDF Team for providing access to advanced data products and is thankful to Audrey Galametz, Dominika Wylezalek, Loredana Vetere, and Roberto Assef for useful discussions and comments on this paper. This work is based on data obtained with the Spitzer Space Telescope, which is operated by the Jet Propulsion Lab (JPL), California Institute of Technology (Caltech), under a contract with NASA. Support was provided by NASA through contract number 1439211 issued by JPL/Caltech. Lawrence Livermore National Laboratory is operated by Lawrence Livermore National Security, LLC, for the U.S. Department of Energy, National Nuclear Security Administration under Contract DE-AC52-07NA27344.

Facilities: Spitzer, UKST

\section{REFERENCES}

Abadi, M. G., Lambas, D. G., \& Muriel, H. 1998, ApJ, 507, 526

Alberts, S., Pope, A., Brodwin, M., et al. 2014, MNRAS, 437, 437

Andreon, S., Trinchieri, G., \& Pizzolato, F. 2011, MNRAS, 412, 2391

Ashby, M. L. N., Stanford, S. A., Brodwin, M., et al. 2013, ApJS, 209, 22

Ashby, M. L. N., Stern, D., Brodwin, M., et al. 2009, ApJ, 701, 428

Austermann, J. E., Aird, K. A., Beall, J. A., et al. 2012, Proc. SPIE, 8452,845239

Bahcall, N. A. 1988, ARA\&A, 26, 631

Bahcall, N. A., Cen, R., \& Gramann, M. 1993, ApJL, 408, L77

Bahcall, N. A., Dong, F., Hao, L., et al. 2003, ApJ, 599, 814

Berlind, A. A., \& Weinberg, D. H. 2002, ApJ, 575, 587

Berlind, A. A., Weinberg, D. H., Benson, A. J., et al. 2003, ApJ, 593, 1

Blakeslee, J. P., Holden, B. P., Franx, M., et al. 2006, ApJ, 644, 30

Bleem, L. E., Standler, B., Brodwin, M., et al. 2014, ApJS, submitted (arXiv:1403.7186)

Borgani, S., Plionis, M., \& Kolokotronis, V. 1999, MNRAS, 305, 866

Brodwin, M., Brown, M. J. I., Ashby, M. L. N., et al. 2006, ApJ, 651, 791

Brodwin, M., Gonzalez, A. H., Moustakas, L. A., et al. 2007, ApJL, 671, L93

Brodwin, M., Gonzalez, A. H., Stanford, S. A., et al. 2012, ApJ, 753, 162

Brodwin, M., Stanford, S. A., Gonzalez, A. H., et al. 2013, ApJ, 779, 138

Brodwin, M., Stern, D., Vikhlinin, A., et al. 2011, ApJ, 732, 33

Bruzual, G., \& Charlot, S. 2003, MNRAS, 344, 1000

Cannon, R. D. 1984, in IAU Colloq. 78, Astronomy with Schmidt-Type

Telescopes, ed. M. Capaccioli (Dordrecht: D. Reidel Publishing), 110, 25

Carlstrom, J. E., Ade, P. A. R., Aird, K. A., et al. 2011, PASP, 123, 568

Collins, C. A., Guzzo, L., Böhringer, H., et al. 2000, MNRAS, 319, 939

Cooper, M. C., Newman, J. A., Coil, A. L., et al. 2007, MNRAS, 376, 1445

Cooray, A., \& Sheth, R. 2002, PhR, 372, 1

Croft, R. A. C., Dalton, G. B., Efstathiou, G., Sutherland, W. J., \& Maddox, S. J. 1997, MNRAS, 291, 305

Demarco, R., Wilson, G., Muzzin, A., et al. 2010, ApJ, 711, 1185

Desai, S., Armstrong, R., Mohr, J. J., et al. 2012, ApJ, 757, 83

Eisenhardt, P. R., Stern, D., Brodwin, M., et al. 2004, ApJS, 154, 48

Eisenhardt, P. R. M., Brodwin, M., Gonzalez, A. H., et al. 2008, ApJ, 684, 905

Elbaz, D., Daddi, E., Le Borgne, D., et al. 2007, A\&A, 468, 33

Elston, R. J., Gonzalez, A. H., McKenzie, E., et al. 2006, ApJ, 639, 816

Fakhouri, O., Ma, C.-P., \& Boylan-Kolchin, M. 2010, MNRAS, 406, 2267

Fassbender, R., Nastasi, A., Böhringer, H., et al. 2011, A\&A, 527, L10

Fazio, G. G., Hora, J. L., Allen, L. E., et al. 2004, ApJS, 154, 10

Fry, J. N., \& Gaztanaga, E. 1993, ApJ, 413, 447

Galametz, A., Stern, D., De Breuck, C., et al. 2012, ApJ, 749, 169

Galametz, A., Stern, D., Pentericci, L., et al. 2013, A\&A, 559, A2

Galametz, A., Stern, D., Stanford, S. A., et al. 2010, A\&A, 516, A101

George, E. M., Ade, P., Aird, K. A., et al. 2012, Proc. SPIE, 8452, 84521

Gettings, D. P., Gonzalez, A. H., Stanford, S. A., et al. 2012, ApJL, 759, L23

Gonzalez, A. H., Stanford, S. A., Brodwin, M., et al. 2012, ApJ, 753, 163

Gonzalez, A. H., Zaritsky, D., \& Wechsler, R. H. 2002, ApJ, 571, 129

Hambly, N. C., MacGillivray, H. T., Read, M. A., et al. 2001, MNRAS, 326,1279

Hamilton, A. J. S. 1993, ApJ, 417, 19

Hartley, M., \& Dawe, J. A. 1981, PASAu, 4, 251
Hasselfield, M., Hilton, M., Marriage, T. A., et al. 2013, JCAP, 7, 8

Hayashi, M., Kodama, T., Koyama, Y., et al. 2010, MNRAS, 402, 1980

Hilton, M., Stanford, S. A., Stott, J. P., et al. 2009, ApJ, 697, 436

Holder, G. P., Viero, M. P., Zahn, O., et al. 2013, ApJL, 771, L16

Huchra, J. P., Henry, J. P., Postman, M., \& Geller, M. J. 1990, ApJ, 365, 66

Jannuzi, B. T., \& Dey, A. 1999, in ASP Conf. Proc. 193, The Hy-Redshift

Universe: Galaxy Formation and Evolution at High Redshift, ed. A. J. Bunker

\& W. J. M. van Breugel (San Francisco, CA: ASP), 258

John, T. L. 1988, A\&A, 193, 189

Kaiser, N. 1984, ApJL, 284, L9

Kitayama, T., \& Suto, Y. 1996, ApJ, 469, 480

Kravtsov, A. V., Berlind, A. A., Wechsler, R. H., et al. 2004, ApJ, 609, 35

Lewis, A., \& Bridle, S. 2002, PhRvD, 66, 103511

Limber, D. N. 1953, ApJ, 117, 134

Lonsdale, C. J., Smith, H. E., Rowan-Robinson, M., et al. 2003, PASP, 115, 897

Ma, C.-P., \& Fry, J. N. 2000, ApJ, 543, 503

Martinez-Manso, C.-P., Gonzalez, A. H., Ashby, M. L. N., et al. 2015, MNRAS, 446, 169

Mei, S., Holden, B. P., Blakeslee, J. P., et al. 2009, ApJ, 690, 42

Mei, S., Stanford, S. A., Holden, B. P., et al. 2012, ApJ, 754, 141

Minkowski, R. L., \& Abell, G. O. 1963, in Basic Astronomical Data: Stars and

Stellar Systems, ed. K. A. Strand (Chicago, IL: Univ. Chicago Press), 481

Mo, H. J., \& White, S. D. M. 1996, MNRAS, 282, 347

Muzzin, A., Marchesini, D., Stefanon, M., et al. 2013a, ApJS, 206, 8

Muzzin, A., Wilson, G., Demarco, R., et al. 2013b, ApJ, 767, 39

Muzzin, A., Wilson, G., Yee, H. K. C., et al. 2012, ApJ, 746, 188

Nantais, J. B., Rettura, A., Lidman, C., et al. 2013, A\&A, 556, A112

Overzier, R. A., Shu, X., Zheng, W., Rettura, A., et al. 2009, ApJ, 704, 548

Papovich, C. 2008, ApJ, 676, 206

Papovich, C., Momcheva, I., Willmer, C. N. A., et al. 2010, ApJ, 716, 1503

Patel, S. G., Holden, B. P., Kelson, D. D., Illingworth, G. D., \& Franx, M. 2009, ApJL, 705, L67

Peacock, J. A., \& Smith, R. E. 2000, MNRAS, 318, 1144

Peacock, J. A., \& West, M. J. 1992, MNRAS, 259, 494

Peebles, P. J. E. 1980, Research supported by the National Science Foundation (Princeton, NJ: Princeton Univ. Press)

Phillipps, S., Fong, R., Fall, R. S. E. S. M., \& MacGillivray, H. T. 1978, MNRAS, 182,673

Pierre, M., Pacaud, F., Juin, J. B., et al. 2011, MNRAS, 414, 1732

Postman, M., Huchra, J. P., \& Geller, M. J. 1992, ApJ, 384, 404

Raichoor, A., Mei, S., Nakata, F., et al. 2011, ApJ, 732, 12

Reichardt, C. L., Stalder, B., Bleem, L. E., et al. 2013, ApJ, 763, 127

Reid, I. N., Brewer, C., Brucato, R. J., et al. 1991, PASP, 103, 661

Rettura, A., Mei, S., Stanford, S. A., et al. 2011, ApJ, 732, 94

Rettura, A., Rosati, P., Nonino, M., et al. 2010, ApJ, 709, 512

Rosati, P., Tozzi, P., Gobat, R., et al. 2009, A\&A, 508, 583

Sawicki, M. 2002, AJ, 124, 3050

Scoccimarro, R., Sheth, R. K., Hui, L., \& Jain, B. 2001, ApJ, 546, 20

Scoville, N., Arnouts, S., Aussel, H., et al. 2013, ApJS, 206, 3

Seljak, U. 2000, MNRAS, 318, 203

Sheth, R. K., Mo, H. J., \& Tormen, G. 2001, MNRAS, 323, 1

Simpson, C., \& Eisenhardt, P. 1999, PASP, 111, 691

Snyder, G. F., Brodwin, M., Mancone, C. M., et al. 2012, ApJ, 756, 114

Sorba, R., \& Sawicki, M. 2010, ApJ, 721, 1056

Spergel, D. N., Verde, L., Peiris, H. V., et al. 2003, ApJS, 148, 175

Springel, V., White, S. D. M., Jenkins, A., et al. 2005, Natur, 435, 629

Stanford, S. A., Brodwin, M., Gonzalez, A. H., et al. 2012, ApJ, 753, 164

Stanford, S. A., Eisenhardt, P. R., Brodwin, M., et al. 2005, ApJL, 634, L129

Stern, D., Eisenhardt, P., Gorjian, V., et al. 2005, ApJ, 631, 163

Stern, D., Jimenez, R., Verde, L., Stanford, S. A., \& Kamionkowski, M 2010, ApJS, 188, 280

Stern, D., Kirkpatrick, J. D., Allen, L. E., et al. 2007, ApJ, 663, 677

Story, K. T., Reichardt, C. L., Hou, Z., et al. 2013, ApJ, 779, 86

Strazzullo, V., Rosati, P., Pannella, M., et al. 2010, A\&A, 524, A17

Tadaki, K.-I., Kodama, T., Ota, K., et al. 2012, MNRAS, 423, 2617

Tinker, J. L., Robertson, B. E., Kravtsov, A. V., et al. 2010, ApJ, 724, 878

Tinker, J. L., Weinberg, D. H., Zheng, Z., \& Zehavi, I. 2005, ApJ, 631, 41

Tran, K.-V. H., Papovich, C., Saintonge, A., et al. 2010, ApJL, 719, L126

Vikhlinin, A., Burenin, R. A., Ebeling, H., et al. 2009, ApJ, 692, 1033

Wang, L., \& Steinhardt, P. J. 1998, ApJ, 508, 483

Wilson, G., Muzzin, A., Yee, H. K. C., et al. 2009, ApJ, 698, 1943

Wylezalek, D., Galametz, A., Stern, D., et al. 2013, ApJ, 769, 79

Younger, J. D., Bahcall, N. A., \& Bode, P. 2005, ApJ, 622, 1

Zeimann, G. R., Stanford, S. A., Brodwin, M., et al. 2012, ApJ, 756, 115

Zeimann, G. R., Stanford, S. A., Brodwin, M., et al. 2013, ApJ, 779, 137

Zheng, Z., Berlind, A. A., Weinberg, D. H., et al. 2005, ApJ, 633, 791 